\title{
A New Mechanism for Mesoscale Legged Locomotion in Compliant Tubular Environments
}

\author{
Pietro Valdastri, Member, IEEE, Robert J. Webster, III, Member, IEEE, Claudio Quaglia, \\ Marco Quirini, Student Member, IEEE, Arianna Menciassi, Member, IEEE, and Paolo Dario, Fellow, IEEE
}

\begin{abstract}
We present design and experimental performance results for a novel mechanism for robotic legged locomotion at the mesoscale (from hundreds of microns to tens of centimeters). The new mechanism is compact and strikes a balance between conflicting design objectives, exhibiting high foot forces and low power consumption. It enables a small robot to traverse a compliant, slippery, tubular environment, even while climbing against gravity. This mechanism is useful for many mesoscale locomotion tasks, including endoscopic capsule robot locomotion in the gastrointestinal tract. It has enabled fabrication of the first legged endoscopic capsule robot whose mechanical components match the dimensions of commercial pill cameras (11 mm diameter by $25 \mathrm{~mm}$ long). A novel slot-follower mechanism driven via lead screw enables the mechanical components of the capsule robot to be as small while simultaneously generating $0.63 \mathrm{~N}$ average propulsive force at each leg tip. In this paper, we describe kinematic and static analyses of the lead screw and slot-follower mechanisms, optimization of design parameters, and experimental design and tuning of a gait suitable for locomotion. A series of ex vivo experiments demonstrate capsule performance and ability to traverse the intestine in a manner suitable for inspection of the colon in a time period equivalent to standard colonoscopy.
\end{abstract}

Index Terms-Biomechatronics, biorobotics, capsular endoscopy, endoluminal surgery, legged locomotion, robotic endoscopy.

\section{INTRODUCTION}

$\mathbf{E}$ NDOLUMINAL devices for minimally invasive surgical and/or diagnostic applications show promise for improving treatment of a number of diseases [1]-[4], particularly in the gastrointestinal (GI) tract [5], [6]. These GI devices have proven

Manuscript received October 21, 2008; revised January 11, 2009. First published March 10, 2009; current version published October 9, 2009. This paper was recommended for publication by Associate Editor H. R. Choi and Editor F. Park upon evaluation of the reviewers' comments. This study was supported in part by the Intelligent Microsystem Center, Korean Institute of Science and Technology (KIST), Korea, in part by the European Commission in the framework of VECTOR FP6 European Project European Union/Information Society Technologies (EU/IST)-2006-033970, as well as the National Science Foundation Graduate Research Fellowship Program (GRFP). Some preliminary results in this paper have been presented at the IEEE International Conference on Robotics and Automation, 2007.

P. Valdastri, C. Quaglia, and M. Quirini are with the Center of Research in Microengineering (CRIM) Laboratory, Scuola Superiore Sant'Anna, Pisa 56025, Italy (e-mail: pietro@sssup.it; c.quaglia@crim.sssup.it; m.quirini@ crim.sssup.it)

R. J. Webster, III is with the Vanderbilt University, Nashville, TN 37235 USA (e-mail: robert.webster@vanderbilt.edu).

A. Menciassi and P. Dario are with the Center of Research in Microengineering (CRIM) Laboratory, Scuola Superiore Sant'Anna, Pisa 56025, Italy. They are also with the Italian Institute of Technology (IIT) Network, 16163 Genova, Italy (e-mail: arianna@sssup.it; dario@sssup.it).

Color versions of one or more of the figures in this paper are available online at http://ieeexplore.ieee.org.

Digital Object Identifier 10.1109/TRO.2009.2014127 particularly clinically beneficial for diagnoses in the small intestine, which is extremely challenging to access without them. However, they currently lack active locomotion, instead relying on natural GI peristalsis - the muscle activity that ordinarily moves food during digestion. Consequently, they cannot stop, control their speed and orientation, or reverse direction. These limitations are particularly problematic once the capsule reaches the large intestine, or colon. Here, capsule diameter is significantly smaller than the lumen in which it operates, so motion is no longer predictable or controllable, making it impossible to fully inspect the interior surface.

This has motivated the recent development of a variety of strategies for locomotion in the colon [7]-[9]. However, to date, all are designed to travel against peristalsis. Furthermore, because they are not swallowable, they do not have the potential to eliminate the hospital visit associated with standard colonoscopy. The ideal system for lower GI access would combine the most beneficial elements of both the pill cameras (i.e., being small enough to swallow and moving in the direction of natural peristalsis), and actively locomoting devices (i.e., the ability to move as desired), while not requiring inflation, which stretches the colon causing pain.

An extensive and detailed review of wireless capsular endoscopy (WCE) is reported in [10], and a focused overview of microrobotic solutions for future GI endoscopy can be found in [11]. To summarize, there are two basic strategies for providing a WCE with active locomotion. One is an external approach where actuation is outside the capsule from, e.g., an external magnetic field (see, e.g., [12] and [13]). The other alternative is a miniaturized locomotion system integrated onboard the capsule (see, e.g., [14] and [15]). For clinical utility, any locomotion strategy must address the specific challenges of the intestine environment as outlined in [16], implying that the capsule must be capable of locomotion on a slippery, elastic substrate. The gut is a compliant, nonlinear, viscoelastic material, typically covered by a thick (up to $2 \mathrm{~mm}$ ) layer of lubricating mucus. It has a coefficient of friction on the order of $10^{-3}$ to $10^{-4}$. Taking into account the guidelines provided by [16], legged locomotion appears to have many advantages [17]. This approach has been investigated intensively by the authors via experiments using a succession of increasingly sophisticated prototypes [18], [19], the most advanced of which is the robot described in this study.

Despite the successes of our earlier prototypes in demonstrating the promise of legged locomotion, a number of limitations remained, which the current prototype is designed to address. These include dimensions incompatible with swallowing, and some difficulty traversing flexures in the intestine, which we 


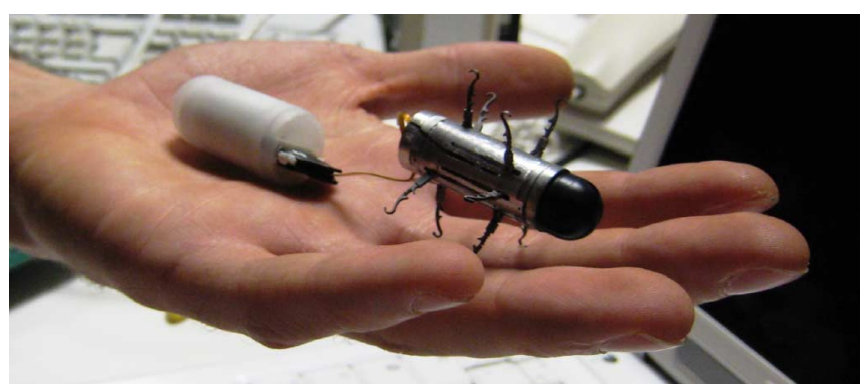

Fig. 1. Prototype of 12-legged endoscopic capsular robot. The plastic rear module contains a battery power supply, and will be optimized and miniaturized in future studies.

hypothesize was due to both capsule size and leg placement. The new design that is the subject of this paper is significantly smaller; its mechanical components now match the dimensions of commercial pill cameras. It also has 12 legs that are axially nearer the center of the capsule to enhance turning. Leg tips, when open, are now also nearly equally radially offset from one another, which aids in tissue distention (improving camera images) and enhances locomotion (providing more evenly distributed points of contact with the intestine). This new 12-leg design, pictured in Fig. 1, features two leg sets (LS) of six legs each. In this paper, we expand upon initial design work reported in [20] and also describe the fabricated prototype and a number of validation experiments using it. This design is an important step toward an eventual pill-based colonoscopy system, mechanically enabling legged locomotion in a pill-sized device for the first time, and including enough legs to uniformly distend collapsed colon tissue, enhancing visualization and locomotion efficiency.

\section{A. Contribution}

Our novel and recently patented [21] slot-follower/lead screw mechanism contributes the state-of-the-art in mesoscale robotic locomotion, providing a compact and powerful means of coupling actuators to sets of legs. We also contribute a tuned gait profile suitable for high-quality locomotion in slippery and deformable environments. In terms of medical capsule robot technology, we contribute a design and fabricated prototype (Fig. 1) that increases the maximum number of legs to 12 , while (perhaps even more importantly) reducing it toward a swallowable size. We also contribute analysis that permits optimization of our mechanism based on design specifications. Experimental contributions include: 1) verification of prototype performance in comparison to design specifications; 2) demonstration that additional legs are useful for uniform distension of deflated colon tissue; 3) determination of gait parameters that enable rapid, efficient, and high-quality locomotion in the GI tract; and 4) assessment of capsule locomotion capability in realistic scenarios similar to conditions that would be encountered in vivo.

\section{DESIGN SPECIFICATIONS AND OVERVIEW}

The size, speed, and safety requirements for any capsule robot are primarily determined by medical considerations. These are obtained from physicians in terms of general objectives. Such objectives can then be taken into account when considering capsule design.

\section{A. Medical Considerations}

Medical considerations for capsule robot design include:

1) Size. Ideally, a capsule robot should be small enough to swallow. However, "swallowable" is somewhat challenging to define, because the maximum swallowable size varies from person to person. However, since commercial pill cameras (e.g., the Given Imaging PillCam which is $11 \mathrm{~mm}$ in diameter and $26 \mathrm{~mm}$ long [22]) have been used in extensive clinical testing, any device that matches their dimensions can be considered swallowable.

2) Speed. A standard colonoscopy is completed in approximately $20 \mathrm{~min}$ to $1 \mathrm{~h} \mathrm{[23].} \mathrm{It} \mathrm{is} \mathrm{desirable} \mathrm{for} \mathrm{a} \mathrm{locomoting}$ robot to travel through the colon in a similar time period.

3) Safety. Contact with the walls of the colon should cause no more damage than a standard colonoscope.

4) Pain reduction. Air insufflation during standard colonoscopy causes abdominal pain for the patient. For this reason, the capsule should have a locomotion system able to propel it without insufflation.

5) Functionality. Physicians must be able to visually observe the interior of the colon. More advanced goals include obtaining biopsies and delivering treatments directly. In this paper, we focus on the basic inspection goal.

\section{B. Design Considerations}

We take the earlier medical objectives into account in determining design specifications for the capsule robot. One design consideration is the number of legs the capsule should include. It seems desirable to maximize the number of legs, since more legs distribute the force needed to propel the capsule over more points of contact, reducing individual foot forces. This will improve propulsion by reducing the impact of single-foot slippage and also make each foot gentler to the colon wall. A larger number of legs will also distend the colon more uniformly and improve visualization of the inner surface. We note that leg design (e.g., knee and foot shape, position, and materials) has been extensively treated in prior works, and refer the interested reader to [18], [19], and [24].

Another important design consideration is actuator selection. Experience in testing and modeling legged capsules [25] indicates that for a 12-legged capsule robot design, as described in the following sections, approximately $2 / 3 \mathrm{~N}$ for each individual foot will be sufficient to propel the capsule along the colon. There is a relatively long lever arm outside the capsule compared to that within, so this force requirement implies that actuators must be both powerful and compact. A dc brushless motor (SBL04-0829, Namiki Precision Jewel, Inc.) was identified in [18] as suitable actuator for similar design requirements on a previous capsule prototype. The motor has an external diameter of $4 \mathrm{~mm}$ and a total length of $16.2 \mathrm{~mm}$ (gearhead included), with a maximum output torque of $0.058 \mathrm{mN} \cdot \mathrm{m}$ before the 79:1 gearhead (PG04-79). 


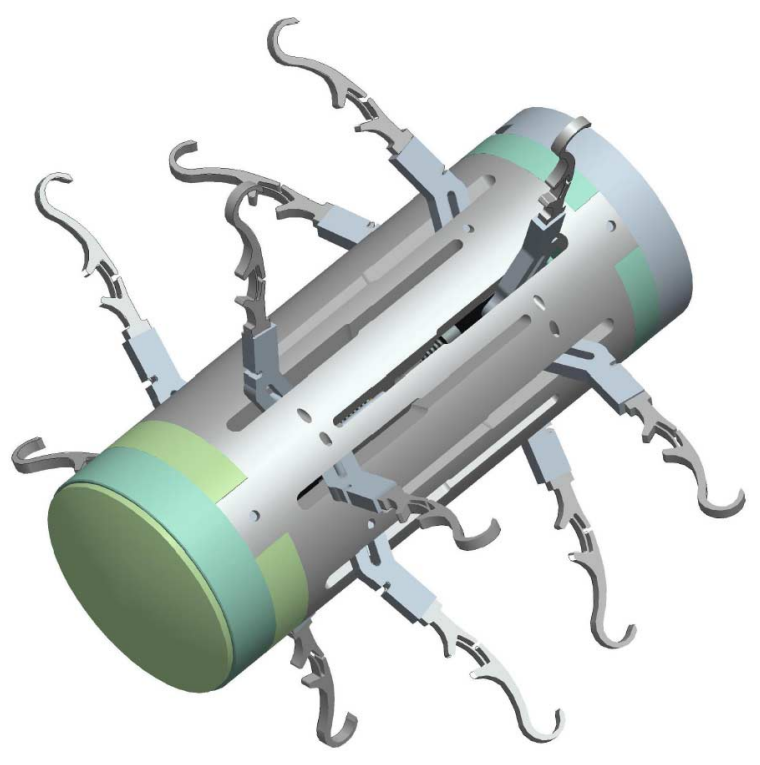

Fig. 2. Isometric view of the 12-legged capsule.

Once the legged concept, number of legs, and actuators have been evaluated, the next set of considerations involve the placement of the legs on the surface of the capsule and the gait that they should utilize. It has been shown that successful locomotion is possible with two LS, one near the front and one near the rear of the capsule [19]. We adopt this general strategy, only we place the LS closer to one another, which is expected to improve the capsule's angular maneuverability. The rear LS has the primary function of producing thrust force, while the front LS is used for the dual purposes of bracing the capsule against unwanted backward motion as rear legs retract and also to help propel the capsule around curves. Fig. 2 illustrates our novel capsule design with its two LS. This choice implies the use of two dc brushless motors, in order to move the two LS independently. Designing capsule gait then involves choosing relative frequencies and phases of the two LS, as described in Section VI-A.

Radial leg tip position must also be considered for optimal locomotion and distention of tissue. Our experimental testing has revealed that legs should open to a diameter of approximately $30-35 \mathrm{~mm}$ to suitably engage the colon wall without damaging it. It seems desirable to place leg tips at equal angular spacings around this circle to improve tissue engagement and locomotion ability of the capsule. We do so, as shown in the end-on view of the capsule in Fig. 3. Fig. 4 shows how we achieve this by angling the legs around the motors within the capsule to engage the nuts. The only exception to equal angular placement of leg tips are the legs farthest from the motors (nearest the horizontal plane in Fig. 3). These four legs were shifted away from the horizontal plane by $4^{\circ}$ each (only $1.1 \mathrm{~mm}$ tip displacement from ideal position), to prevent them from interfering with one another when folded into the capsule.

Fig. 4 illustrates the internal mechanics of the capsule. Each motor actuates one set of legs by driving a gear attached to a miniature lead screw. As the screw spins, it translates the nut

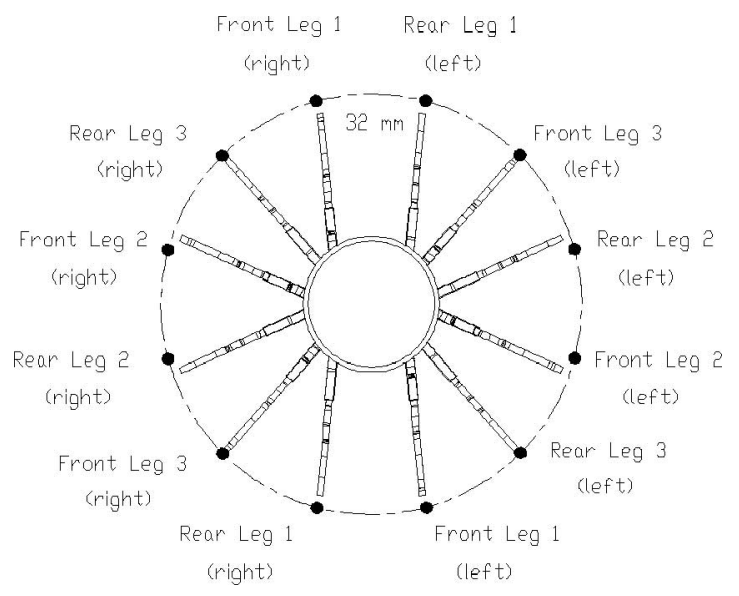

Fig. 3. Front view of the capsule showing a 32-mm-diameter circle, which is suitable for engaging and distending-but not perforating-the colon wall. Note that the 12 leg tips are approximately evenly distributed around the circle.

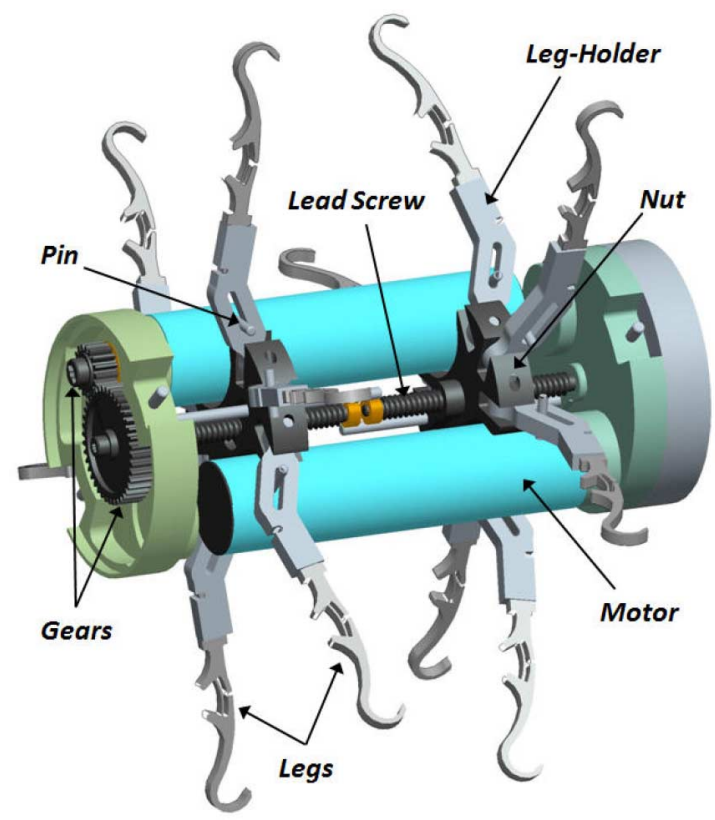

Fig. 4. Side view of the capsule with the capsule body hidden to reveal internal components.

linearly. A leg holder (see Fig. 5) is fixed to the nut with a pin that permits it to rotate as the nut moves. At the capsule wall is another pin, inserted into a slot in the leg holder (this is the slotfollower part of the mechanism). As the nut translates axially up and down along the lead screw, the set of legs makes a stride. All six legs at a given end of the capsule are attached to one nut, and all simultaneously open and close as the nut translates.

\section{KinemATIC ANALYSIS AND OptimiZATION}

While there are many constraints to consider when designing the slot-follower mechanism, there remain a family of possible solutions for link lengths, as well as some freedom of leg placement on the capsule exterior.

We present a solution here that strikes a balance between competing design objectives (e.g., force capability at leg tips 


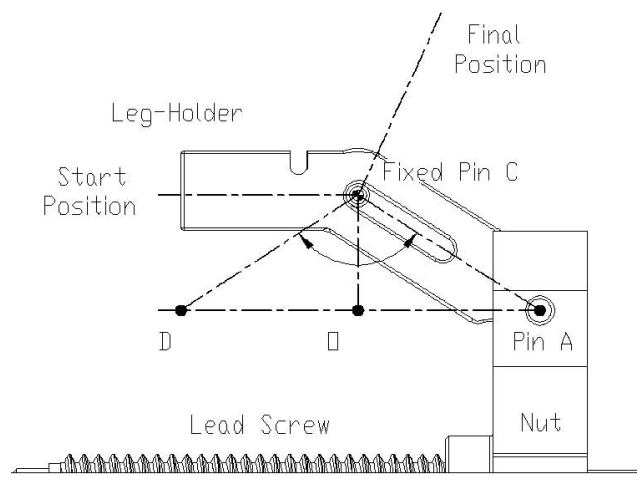

Fig. 5. Side view of the slot-follower mechanism showing its design parameters.

versus overall screw length), while optimizing leg opening angles. However, as will be discussed in Section VIII, we note that optimization of the mechanism for other design objectives is also possible. The design constraints for our capsule robot are as follows:

1) The overall size of the locomotion unit must be at most $11 \mathrm{~mm}$ diameter by $26 \mathrm{~mm}$ in length, matching the size of commercial pill cameras to ensure swallowability.

2) Leg opening angles must be at least $110^{\circ}$ to permit good contact with the colon wall [19].

3) The maximum force at each foot should be approximately $2 / 3 \mathrm{~N}$ as indicated by the aggregate pulling force noted in [25], meaning that the pins in the leg holder must be sufficiently far apart.

4) Legs must all simultaneously retract within the capsule when in the closed position so that the capsule can be swallowed.

5) All components must be sufficiently large and strong enough to withstand the forces they will experience.

6) The capsule should have two degrees of freedom (two independent LS - to enable our general locomotion strategy), and thus must contain two motors.

Fig. 5 shows the geometry of the slot-follower mechanism. While each of the two nuts (one at each end of the capsule) hold six legs, the dimensions of only three on one side of the nut need to be designed because the other three on the opposite side are identical copies of the first three, rotated by $180^{\circ}$ about the central lead screw axis. The first design decision is the length of $\overline{O C_{i}}, i \in\{1,2,3\}$, because many of the aforementioned constraints influence it. While constraint 3 ) provides a test for minimum possible lengths of $\overline{O C_{i}}$, it is generally desirable to make each $\overline{O C_{i}}$ as long as possible to reduce mechanism internal forces. Too long, however, and constraint 2) is violated (as will be further described shortly). In the presence of size constraints 1) and 5), we choose the longest feasible lengths for the $\overline{O C_{i}}$ values and proceed with further analysis using these fixed values.

The $\overline{A D}$ distance will be the same for all legs at a given end of the capsule, because all are attached to the same nut. The maximum possible $\overline{A D}$ distance, fixed by constraints 1) and 5), is approximately $6.2 \mathrm{~mm}$. The $\overline{O D_{i}}$ distances may be selected to

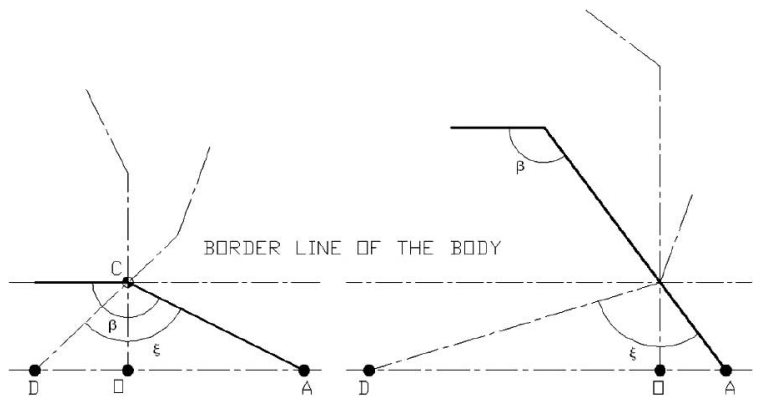

(a)

(b)

Fig. 6. (a) With $\overline{O D} \leq \overline{A D} / 2$, the leg will fold completely inside the capsule body, as indicated by the dark line. (b) If $\overline{O D}>\overline{A D} / 2$ the leg will not close fully within the capsule.

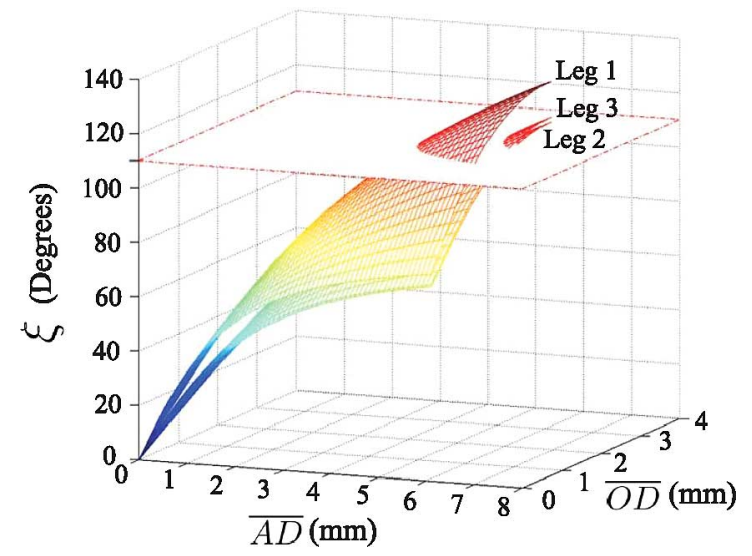

Fig. 7. This plot shows the permissible $\overline{A D}$ and $\overline{O D_{i}}$ combinations, providing a design tool for both leg opening angles and leg placement positions on the outside of the capsule body. The points above the plane that lie on the surfaces do not violate constraint 2). Additionally, all three legs must share a common $\overline{A D}$ (total nut travel) dimension.

place the legs at desired positions on the outside of the capsule, but $\overline{O D_{i}}$ must be no more than $\overline{A D} / 2$, as illustrated in Fig. 6 .

The values $\overline{A D_{i}}, \overline{O D_{i}}, \overline{O C_{i}}$, and $\xi_{i}$ are related by the law of cosines

$$
\xi=\cos ^{-1}\left(\frac{\overline{A D} \cdot \overline{O D}-\overline{O C}^{2}-\overline{O D}^{2}}{\sqrt{\left((\overline{A D}-\overline{O D})^{2}+\overline{O C}^{2}\right)} \sqrt{\left(\overline{O D}^{2}+\overline{O C}^{2}\right)}}\right)
$$

Fig. 7 explores permissible $\overline{A D}$ and $\overline{O D_{i}}$ combinations. The regions above the plane do not violate constraint 2 ), and any set of points that share a common $\overline{A D}$, are on the three surfaces, and are above the plane may be used to select $\overline{O D_{i}}$ values. Selection of $\overline{O D_{i}}$ is equivalent to selecting the position on the outside of the capsule where the legs will be attached. We choose them to maximize the angle to which the legs can open $\left(\xi_{i}\right)$, meaning $\overline{O D_{i}}=\overline{A D} / 2$. All the resulting calculated values are listed in Table I.

\section{Force ANalysis AND LEAD ScRew DESIGN}

The actuation mechanism described earlier was dimensioned in conjunction with a consideration of the force requirements 
TABLE I

PARAMETERS OF SLOT-FOLLOWER MECHANISM

\begin{tabular}{|l||l|l|l|}
\hline & Leg 1 & Leg 2 & $\operatorname{Leg~3}$ \\
\hline$\overline{A D}[\mathrm{~mm}]$ & 6.20 & 6.20 & 6.20 \\
\hline$\overline{O C}[\mathrm{~mm}]$ & 2.03 & 1.50 & 1.96 \\
\hline$\overline{O D}[\mathrm{~mm}]$ & 3.10 & 3.10 & 3.10 \\
\hline$\xi\left[^{\circ}\right]$ & 113.57 & 128.37 & 115.32 \\
\hline$\beta\left[^{\circ}\right]$ & 146.78 & 154.19 & 147.66 \\
\hline
\end{tabular}

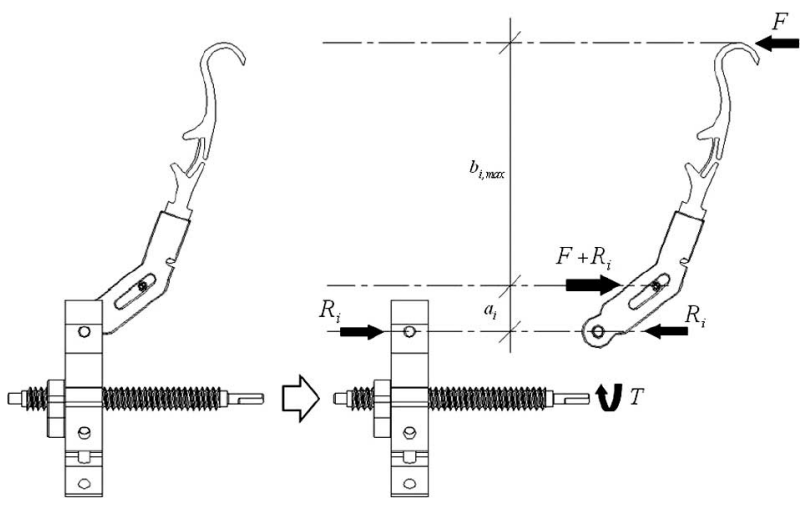

Fig. 8. Free-body diagram of the mechanism showing forces and torques.

at the leg tips. Here, we provide an analysis of the forces and torques in all parts of the lead screw/slot-follower mechanism, to ensure that the actuators are capable of providing the desired foot forces $F$.

\section{A. Converting Foot Force to Lead Screw Force}

As mentioned previously, we estimate $F=2 / 3 \mathrm{~N} / \mathrm{ft}$ as the desired force at each foot. Each foot force produces a reaction force at the nut (amplified by the lever between the pins, as shown in Fig. 8) of

$$
R_{i}=F \frac{b_{i, \max }}{a_{i}}, \quad i \in\{1,2,3\} .
$$

The values $b_{i, \max }$ are the maximum possible lever arms between the pin at the capsule wall and the tip of the leg. The value of $b_{i, \max }$, as well as the position of the nut when it occurs are somewhat involved calculations, and are detailed in the Appendix of [20]. For current purposes, it is enough to say that they will be in the range of 11.7-12.2 $\mathrm{mm}$, depending on the leg.

The summation of these six reaction forces on the nut

$$
W=2 \sum_{i=1}^{3}\left(R_{i}\right)
$$

is the total linear force that the lead screw must provide when the legs are in their worst-case configurations and simultaneously loaded with maximum foot forces. Note that this configuration is not quite physically realizable, since the legs open at different rates due to the different $\overline{O C}$ distances. Therefore, all feet cannot simultaneously achieve their longest possible lever arms. However, considering the law of cosines given in Section III with (2) as written, the calculation is conservative. It will yield
TABLE II

DESIGN PARAMETERS OF THE LEAD SCREW

\begin{tabular}{|l|l||l|l|}
\hline$T[\mathrm{mNm}]$ & 3.64 & $d_{m}[\mathrm{~mm}]$ & 1.00 \\
\hline$R_{1}[N]$ & 3.59 & $L[\mathrm{~mm}]$ & 0.25 \\
\hline$R_{2}[N]$ & 5.07 & $\alpha_{n}\left[^{\circ}\right]$ & 14.44 \\
\hline$R_{3}[N]$ & 3.75 & $\mu$ & 0.25 \\
\hline$W[N]$ & 24.81 & & \\
\hline
\end{tabular}

a slightly higher torque required from the actuators than will be required in the worst-case physically realizable configuration.

The torque that must be applied to the lead screw to overcome $W$ is given by the standard lead screw equation

$$
T=\frac{W d_{m}}{2} \frac{\mu \pi d_{m}+L \cos \alpha_{n}}{\pi d_{m} \cos \alpha_{n}-\mu L}
$$

where $d_{m}$ is the pitch diameter of the screw, $\mu$ is the coefficient of friction between the lead screw and the nut, $\alpha_{n}$ is the crosssection angle of the thread (measured in a plane perpendicular to the helical profile of the screw), and $L$ is the axial pitch of the screw. The lead screw is connected to the dc brushless motor through a gear reduction with a ratio of 0.425, as shown in Fig. 4 .

\section{B. Lead Screw Design}

The lead screw has three parameters that may be designed to meet the device specifications: the pitch diameter $d_{m}$, the axial pitch $L$, and the coefficient of friction $\mu$. It is desirable to use standard screw sizes to simplify manufacture of the nut, since it is possible to purchase standard taps to cut the internal threads of the nut for standard screw sizes. Each standard $d_{m}$ size has a corresponding pitch. The coefficient of friction can be designed by choosing appropriate materials for the nut and lead screw. For example, a steel-steel surface has a coefficient of 0.12-0.42, depending on lubrication [26].

As the best possible tradeoff between size, speed, and force, we selected a 1 -mm-diameter lead screw $\left(d_{m}=1 \mathrm{~mm}\right)$, with the standard pitch of $0.25 \mathrm{~mm}$ per turn. To minimize wear and increase operative lifetime of the mechanism, steel was chosen as material for both the nut and the lead screw. All the selected design parameters and resulting calculated values for our capsule are listed in Table II.

Under unloaded conditions, the motor can drive the nut with a theoretical speed of $36 \mathrm{~mm} / \mathrm{min}$. Since one opening and closing cycle requires two travels of the nut over its $6.2 \mathrm{~mm}$ range, one unloaded leg cycle can be completed in approximately $21 \mathrm{~s}$. Under full load ( $0.66 \mathrm{~N}$ per leg), the legs will move rather slowly, at $17.25 \mathrm{~mm} / \mathrm{min}$. In this case, one leg cycle can be completed in approximately $43 \mathrm{~s}$. However, the legs are never expected to simultaneously reach full load, even when engaging the colon wall, and there will be large portions of their duty cycle when $b$ is small or there is no foot-tissue contact. Thus, the average foot force over the full stride is far less than $0.66 \mathrm{~N}$, meaning that the nut should ordinarily be capable of moving at higher speeds. Note also that all calculations in this paper are based on considering rigid legs, and will be conservative if flexible legs are used. The legs of the capsule prototype are flexible (made of nitinol, an alloy of nickel and titanium, as 
in [18]), and are provided with a flexure-based joint at the knee. We note that our estimate of required foot forces takes both leg flexibility and the knee joint into account, since it is based on experimentation with similar jointed legs, and both prior and current experimental studies show that these legs do not interfere with the capsule's ability to locomote. The flexibility of the knee can only be beneficial, making our all calculations conservative and reducing all internal mechanism forces calculated in this paper, further improving capsule speed.

\section{FABRICATION}

The 12-legged capsule design, as described in the previous sections, required machining and assembly of more than 70 high-precision components. Almost all these components were fabricated in-house using a microwire electrical discharge machine (EDM) (AP 200L, Sodick, Japan), a sink EDM (Micro Sink, Sarix, Switzerland), and a five-axis micro-CNC machining center (HSPC, KERN GmbH, Germany).

Capsule components were fabricated using these machines as follows:

1) Capsule body. Ergal, an aluminum alloy, was selected as the fabrication material for the capsule body in order to obtain a precise and solid structure. We note however, that polymeric materials, such as polyether ether ketone (PEEK), could be considered in future capsules as an alternative, enhancing biocompatibility. Final dimensions of capsule body were $25 \mathrm{~mm}$ in length and $11 \mathrm{~mm}$ in diameter, as designed. The capsule body was fabricated using the micro-CNC.

2) Legs. For adaptability to the environment, legs must withstand a significant local deformation without failure. Thus, we used a superelastic nitinol to fabricate legs, and each was equipped with a flexible knee $(0.07 \mathrm{~mm}$ in thickness). During leg opening, each knee flexes, adapting to different diameters (ridges) within the colon. The hooked round tip is able to approach the tissue without damage to the mucosa, as demonstrated by in vivo test results reported in [19]. Furthermore, a detailed analysis and optimization of leg design for legged locomotion inside the human bowel is reported in [24]. Legs were fabricated from sheets of nitinol using the microwire EDM described earlier. These parts could also have been fabricated with a laser cutting technique.

3) Leg holders. Each leg snaps into a rectangular groove in its leg holder. Since leg holders require a high level of precision, strength, and wear resistance, they were fabricated from steel using both the EDM machines listed earlier. Alternate techniques were not applicable in this case, since the leg holders have complex internal geometry.

4) Gears. The gears that couple the motor shafts to the lead screws were made from bronze to achieve low friction, and profiles were cut with a microwire EDM. A microwire EDM or a gear hob can be used to fabricate these parts. Two commercial steel lead screws were modified by microwire EDM to create a flat protrusion at one end that was inserted into the gear.

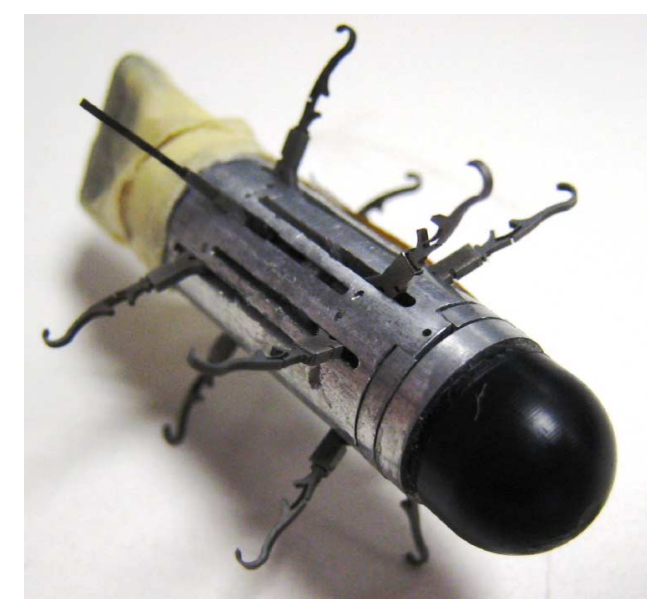

Fig. 9. Assembled 12-legged capsule. The black dome simulates the volume of a snap-on vision system for capsular endoscopy.

5) Nuts. The nuts that travel along the lead screws and apply forces to the leg holders have complex shapes. Due to their complexity, they were fabricated in a multistep procedure involving all of the machining techniques listed earlier.

6) Caps. Four caps were fabricated using the micro-CNC. Two of them are used to fix the position of the motors relative to the screws, and ensure proper gear positions. The other two, placed at the ends of the capsule, are designed to accommodate control electronics.

The assembled device is shown in Fig. 9. All the water- and moisture-sensitive components, such as electronic circuitry and connectors, were sealed inside proper housings in the capsule by using bicomponent epoxy glue. Proper waterproofing was assessed by immersing and operating the capsule in fresh water.

\section{CONTROL SYSTEM}

The capsule uses an open loop motion coordination scheme defined by several gait parameters to determine desired leg opening angles as a function of time. The actual leg opening angles are then servoed to the desired leg opening angles using closedloop low-level motor control. We begin by describing the basic gait, and then discuss implementation.

\section{A. Gait Description}

In order to propel a legged capsule equipped with two independent LS, we defined a gait cycle. A typical example is shown in Fig. 10. In this gait cycle, rear legs are primarily responsible for propulsion, while front legs are primarily responsible for bracing the capsule against backward motion. Here, $\lambda_{1}$ and $\lambda_{2}$ are the average initial angles of the front and rear LS, respectively, measured from the capsule body at the beginning and end of a stride. The angles $\alpha_{1}$ and $\alpha_{2}$ are the average angles of the front and rear LS, respectively, measured from $\lambda_{1}$ and $\lambda_{2}$.

Note that when the offset angles $\lambda_{1}$ and $\lambda_{2}$ are not zero, legs will not close completely. This can be used to reduce stride time, 


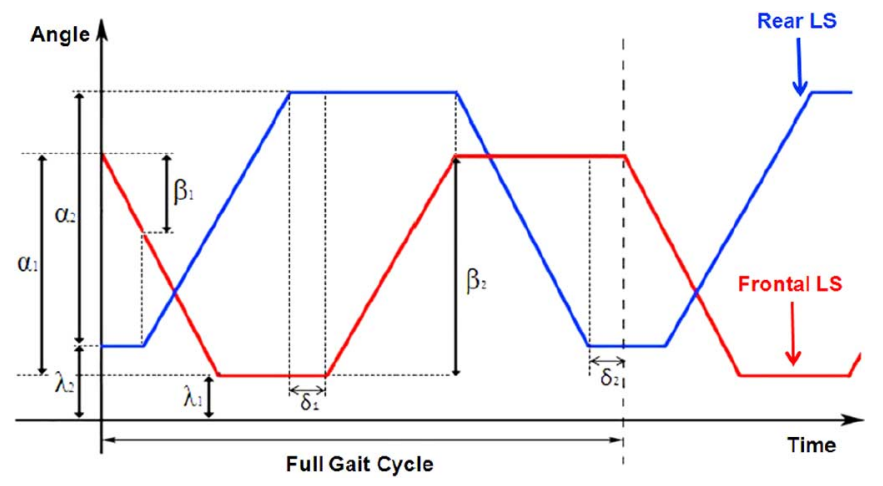

Fig. 10. A full locomotion gait cycle.

and helps prevent tissue from becoming caught underneath the legs as they close.

A second group of parameters shown in Fig. 10 define delays between LS activations. In particular, the $\delta$ parameters define the time delays between the end of the rear LS action (either opening or closing) and the start of the same action by the front LS. Regarding the activation of the rear LS, we found it more straightforward to work with angular rather than time delays. However, the two are approximately equivalent due to closedloop control. Thus, the rear LS starts its action (either opening or closing) as soon as the front LS reaches the $\beta$ angular value. More precisely, $\delta_{1}$ is the time delay between the end of rear LS opening and the beginning of front LS opening, $\delta_{2}$ is the delay between the end of rear LS closing and the beginning of front LS closing, $\beta_{1}$ is the angular difference between the front LS closing and the beginning of rear LS opening (i.e., the rear LS starts opening as soon as the front LS closes to $\beta_{1}$ ), and $\beta_{2}$ is the angular difference between the front LS opening and the beginning of rear LS closing.

Time delays $\delta_{1}$ and $\delta_{2}$ are important to guarantee tissue stabilization around the capsule, maximizing the effect of following actions (anchoring or propulsion), as will be further discussed in Section VII. The angular differences $\beta_{1}$ and $\beta_{2}$ are related to LS synchronization, and their contribution to gait efficiency is assessed experimentally, as described in Section VII.

\section{B. Control Implementation}

The onboard control electronics are based on a double microcontroller architecture that implements a back electromagnetic force strategy for driving the two brushless motors [27]. The first microcontroller is a CC2430 (Texas Instruments, Inc.), enabling wireless bidirectional communication with the usercontrol interface. This device receives the gait parameters and drives one motor through a custom MOSFET driver. The second motor is driven by a C8051F311 microcontroller (Silicon Labs, Inc.) that receives input from a synchronous serial link to the CC2430. Details of this circuitry are reported in [28]. All electronic components were placed on two circular circuit boards with a diameter of $9.7 \mathrm{~mm}$ and a thickness of $2.35 \mathrm{~mm}$. These boards were placed inside the caps on the two ends of the capsule.

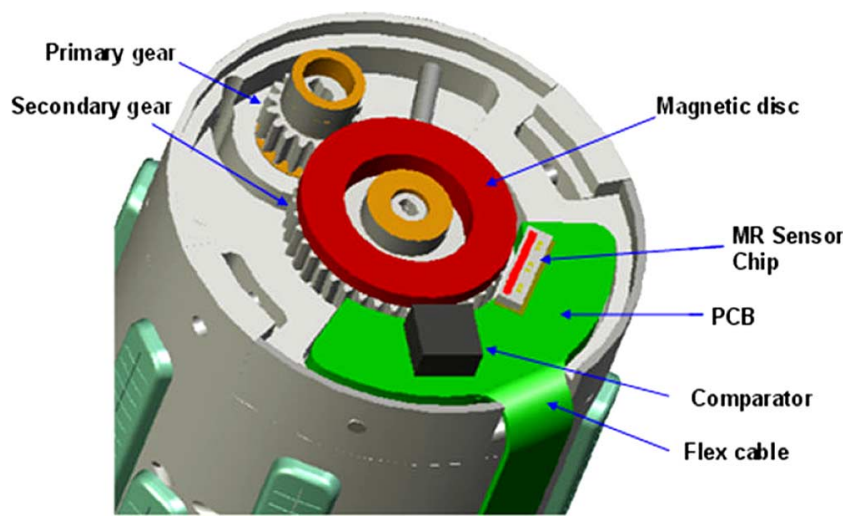

Fig. 11. Computer-aided design (CAD) of the magnetic encoder integrated onboard the 12-legged capsule.

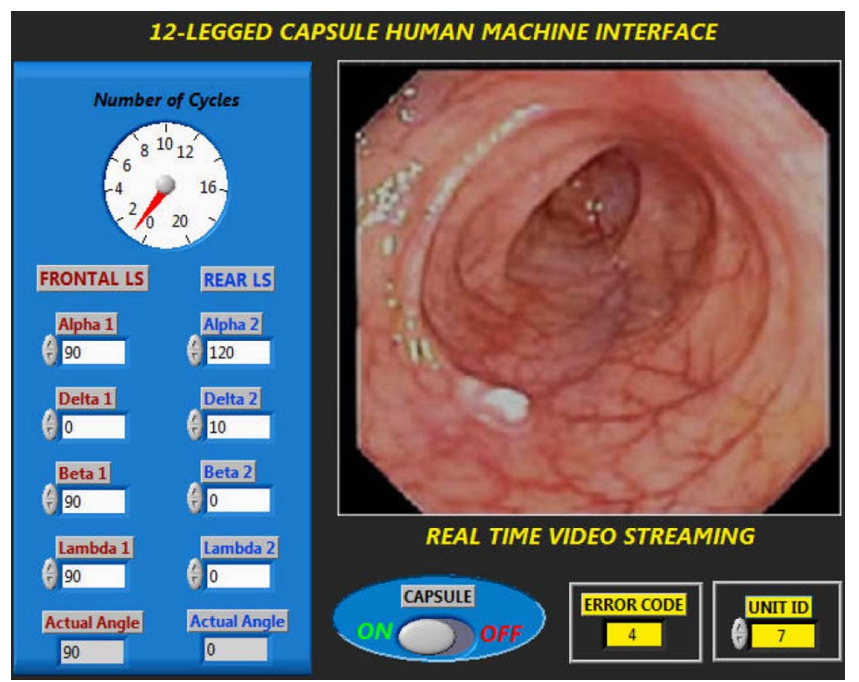

Fig. 12. Snapshot of the human-machine interface for capsule control.

Closed-loop control of both LS was enabled by the integration of two magnetic encoders developed by Sensitec GmbH. The single sensing system consisted of a ring micromachined from $\mathrm{NdFeB}$, magnetized with 16 poles and glued on the secondary gear, as represented in Fig. 11. A pole-fitted anisotropic magnetoresistive (AMR) sensor was mounted on a printed circuit board and placed close to the magnetic ring. In combination with a comparator, rectangular signals were generated at the sensor output that was connected to a counter input of the microcontroller driving the motor connected to the primary gear. A resolution of $5.6^{\circ}$ for the rotation of the secondary gear was obtained. Both LS were equipped with this sensing system.

A human-machine interface (HMI), represented in Fig. 12 was developed using Labview 8.2 (National Instruments). This allows the user to input gait parameters, as defined in Section VI-A, send them to the capsule via the telemetry link, and obtain feedback from the magnetic encoders. The HMI is designed to include real-time streaming image display, as shown in Fig. 12, that will come from a camera attached to the front of the capsule (this camera had not yet been integrated for the experiments described in this paper, but will be similar to the 
cameras used on prior capsules, e.g., [19]). Finally, an error message dialog box enabled feedback to the user in case of malfunctions. The communication between the HMI, running on a standard PC, and the capsule was achieved by a purposely developed dongle, bridging the telemetric link and a universal serial bus (USB) port of the PC. The electrical components of the dongle are a USB/UART converter (UM232R, FTDI Chip, U.K.) and a CC2430 wireless microcontroller.

\section{EXPERIMENTAL RESULTS}

\section{A. Bench Testing}

A first set of experiments was designed to verify that the capabilities of the prototype satisfied design specifications. We first measured the maximum opening angles of each leg and compared them to the design objectives reported in Table I. All measured angles were within $\pm 2^{\circ}$ of design objectives, confirming the effectiveness of fabrication and assembly. We then experimentally determined the time required for a full cycle (opening and closing) of one unloaded LS to be $23 \mathrm{~s}$, which was in close agreement with the theoretical value of $21 \mathrm{~s}$ reported in Section IV-B. Lastly, using a load cell (FMI-210B5, Allurius, Germany) we measured the maximum pulling force that a single LS can produce to be $3.8 \mathrm{~N}$. This implies an average force of $0.63 \mathrm{~N}$ per leg, which is in close agreement with the 2/3 N estimate used in design. Further experiments described below demonstrate that the values measured here are sufficient for propulsion.

\section{B. Closed, Straight Phantom Model Trials}

A second set of experiments designed to evaluate the locomotion ability of the capsule was then performed using a closed, straight ex vivo model. The experimental setup was the same as that used to evaluate previous legged capsule prototypes in [18] and [19], namely a fixture capable of holding a tubular structure such as the gut at both ends. Freshly excised porcine colon specimens were obtained in compliance with standard medical and ethical guidelines from a $50 \mathrm{~kg}$ pig, thus presenting an average diameter similar to that of human beings [29]. The tissue samples were stored in a refrigerated isoosmotic physiological solution to preserve tissue structure and surface properties before testing. To maintain tissue hydration during testing, the colon was hydrated with $1 \mathrm{ml}$ of the solution every 3-5 min, with excess fluid blotted away. Furthermore, to rule out the effects of tissue degradation over time, testing time for evaluation of speed and thrust force was limited to 15-20 min from start to end per sample.

The colon specimen was fixed at both ends of the testbed, as shown in Fig. 13(a). This setup also made it possible to adjust the path that the capsule traverses by changing the height and the distance between the fixtures. We used a configuration where the colon was freely suspended with its lumen collapsed. These tests were performed with a wired power supply.

Using this setup, we conducted an experiment to compare tissue distension capabilities of the capsule against those of our previous eight-legged design [19]. As described in Section II-B,
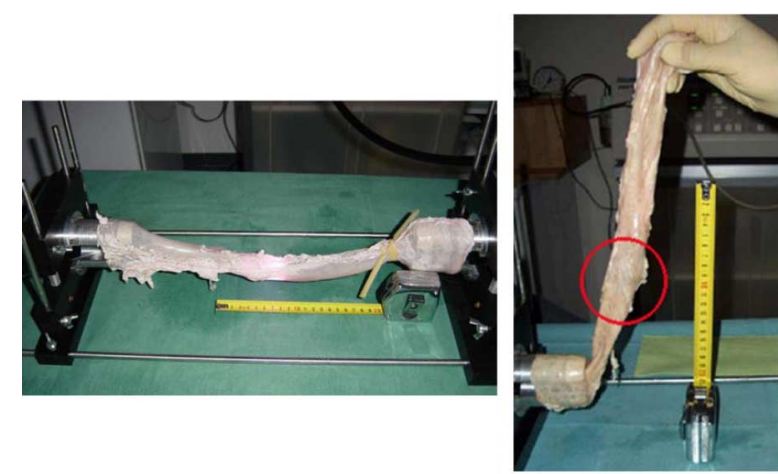

(a)

(b)

Fig. 13. Experimental apparatus used for (a) straight and (b) vertical ex vivo experiments.

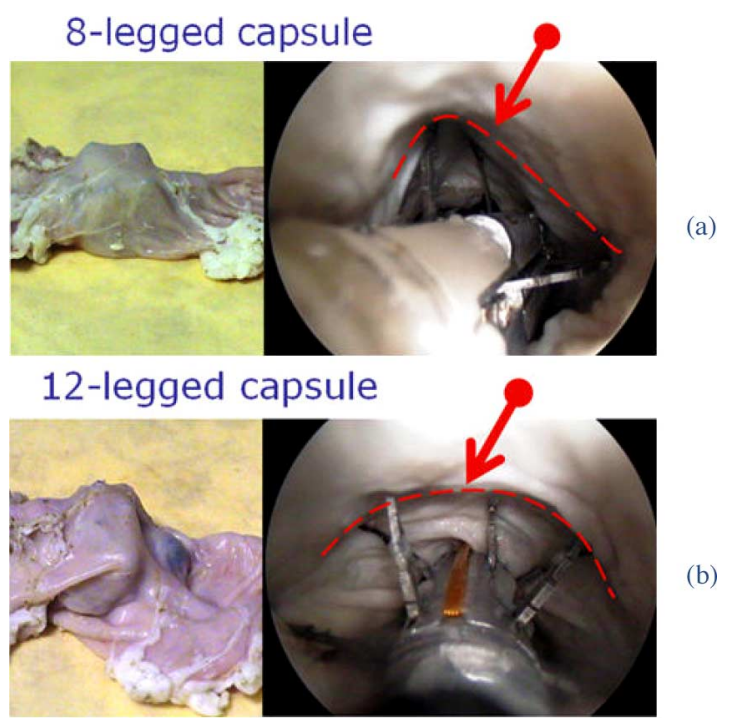

Fig. 14. Evaluation of colon wall distension when (a) 4 or (b) 6 legs are included in a single LS. External views of the capsules in the colon are shown in the left images, while endoscopic views of the capsules inside the lumen are shown on the right.

increasing the number of legs and modifying their position so that LS do not align axially (see Fig. 3), distributes the radial forces more evenly, thus reducing the potential for foot slippage and minimizing possible irritation to the lumen wall. As is clearly visible in Fig. 14, the 12-legged capsule [Fig. 14(b)] distended the colon wall in a more uniform manner than the previous eight-legged capsule [Fig. 14(a)].

We then used the straight phantom model to determine a suitable gait for the capsule. Through extensive experimentation with many gait profiles, we obtained the gait represented in Fig. 10. This was due to the following considerations:

1) At the beginning of the gait, front legs should open to center the capsule in the lumen and to firmly attach it to the tissue.

2) The rear legs should open while the front legs are closing for immediate, effective propulsion.

3) When the rear legs are fully open and before they begin to close, the front legs should be fully open in order to brace 
the capsule against backward motion. For this reason, we set $\beta_{2}=\alpha_{1}$.

4) Immediately after the rear legs finish opening, a time delay $\left(\delta_{1}\right)$ is useful to stabilize the capsule inside the lumen. This enables reliable anchoring of the rear LS before the front LS begins to open, preventing backward motion. However, this delay should be as short as possible, since it also increases total gait time.

5) Similarly a delay $\left(\delta_{2}\right)$ is useful for stabilization after closure of the rear legs and before front legs begin to close, improving propulsion.

After determining this gait profile, we simultaneously tuned all eight different gait parameters described in Section VI-A experimentally. Gait effectiveness was evaluated not only in terms of maximum capsule speed, but also in terms of quality of motion, e.g., centering the capsule in the gut, minimizing potential leg failure, minimizing the potential for tissue entrapment, and uniformity of motion. While a higher speed is desirable in order to reduce the total time of the medical procedure, quality of motion is important for ensuring both safety for the patient and effectiveness of diagnostic results. All tests were performed five times in order to obtain statistical relevance.

Based on the earlier considerations, we experimentally determined gait parameters of $\alpha_{1}=80^{\circ}, \alpha_{2}=110^{\circ}, \lambda_{1}=40^{\circ}, \lambda_{2}=$ $40^{\circ}, \delta_{1}=0.5 \mathrm{~s}, \delta_{2}=0.5 \mathrm{~s}, \beta_{1}=30^{\circ}$, and $\beta_{2}=80^{\circ}$.

With a similar setup, the maximum pulling force of the capsule in the intestine was measured. The load cell described previously was connected to the back of the capsule with a wire through a spring. The recorded value, which depends mostly on the friction between the legs and the colon wall, was $0.2 \mathrm{~N}$.

Vertical locomotion was also demonstrated using the closed straight phantom model. The capsule was inserted into a vertical, loose colon, and net propulsion against gravity was observed, in the manner illustrated in Fig. 13(b).

\section{Lower GI Phantom Model Trials}

A third set of experiments was carried out using a lower GI phantom model, consisting of an anatomical model of the abdominal, chest, and pelvic cavities, with additional accessories for the simulation of organs (e.g., liver, spleen, and sphincter). In addition, the model has fixtures aligned in the shape of human mesentery for the attachment of ex vivo animal intestine. Fresh porcine colon, obtained and preserved, as described in the previous section, was attached alongside the fixtures. Once fixed, the colon can be set up to simulate typical anatomical characteristics, such as the angles and alignment of the sigmoid curve and the sharpness of the left colonic flexure. We used the human-like large bowel geometry model to verify the capsule capability in realistic human-like conditions, as represented on the left side of Fig. 15. The locomotion parameters were set according to the results of the best performance found earlier.

The capsule was able to propel itself through all the parts of the lower GI phantom model, including the hepatic and splenic flexures, thus demonstrating improved capability over the previous eight-legged capsule prototype [19]. The capsule crawled with an average speed of $5 \mathrm{~cm} / \mathrm{min}$. Considering a mean length

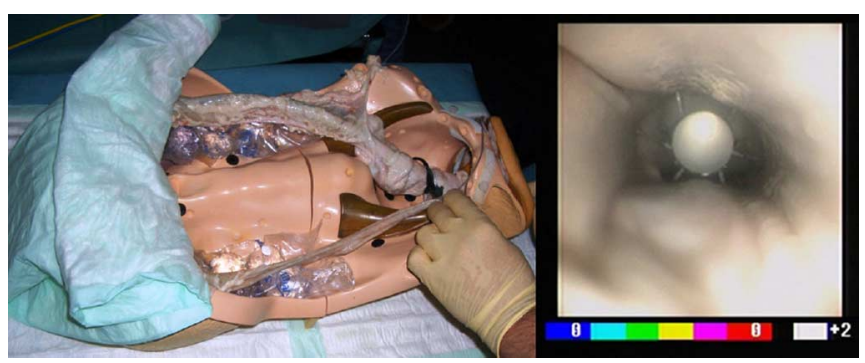

Fig. 15. Illustration of the lower GI phantom model (left) and endoscopic view of the capsule during locomotion in the colon (right).

of $140 \mathrm{~cm}$ for the entire colon, a full passage would take less than $30 \mathrm{~min}$, and is thus within the time frame of a standard colonoscopy. An endoscopic picture of the capsule crawling in the colon can be seen on the right-hand side of Fig. 15.

\section{DISCUSSION}

There are several features of the capsule and lead screw/slotfollower design that merit additional discussion. For example, we anticipate that in addition to distending tissue more uniformly, the number and placement of legs (LS closer to one another and offset radially, as shown in Fig. 3) on the 12-legged capsule will enable it to navigate sharp corners like the colon's splenic flexure, which has been challenging for prior prototypes. We leave verification of this expectation to future in vivo studies.

Another noteworthy property of the lead screw/slot-follower design is that in principle, it allows novel footfall patterns to be created within a single stride. Each leg in a single LS may have a different length, $\overline{O C}$ dimension, and $\overline{O D}$ dimension. These differences permit legs in a single LS to open with different velocities and to different angles, even though they share a common actuator. The analyses in this paper provide a design space under which this may be exploited to create desired footfall patterns. However, we note that drastically different footfall patterns may be impractical for a capsule as small as ours, because there is little flexibility in $\overline{O C}$ dimensions, it is desirable for the overall capsule to remain short (meaning that $\overline{A D}$ should be as short as possible), and legs should open to a maximum angle greater than $90^{\circ}$ to engage the colon wall. However, if the design were scaled up or down in the future for a different application, such footfall pattern flexibility may be useful.

We also note that the mechanical module of the capsule alone is $25 \mathrm{~mm}$ in length in our design, and the length of the capsule is $29 \mathrm{~mm}$ when the end caps and electronics they house are included. If we also consider the volume required by a snap-on vision system for capsular endoscopy, typically $300 \mathrm{~mm}^{3}$, the total length will be $33 \mathrm{~mm}$. At these dimensions, it is likely that only a certain percentage of the population will be able to swallow the complete device. Thus, while our capsule represents a significant volume and length reduction compared to prior designs, some further miniaturization is needed before robotic capsules are generally applicable for all throat sizes. An alternative (since the throat is the limiting factor in terms of capsule size) is to swallow the capsule in several pieces (e.g., motor module, camera module, electronics module, power module) 
that assemble in the stomach to form a complete capsule robot. This concept is similar to an ongoing effort to create assembling reconfigurable endoluminal surgical systems [30].

Another area of potential future research is in gait design. The locomotion gait used in this paper is essentially open-loop motion coordination, where time and angular delays, namely $\delta$ and $\beta$, account for dynamic effects in the gait, allowing them to die out before the next step. A dynamic model of foot-colon interaction has the potential to improve capsule locomotion, and will be studied in future work.

Finally, power is an important consideration for a capsule robot such as ours. For the experiments described in this paper, the capsule was connected to external power using wires. Eliminating them is an important challenge in future capsule robot design. Average power required for locomotion of our capsule was experimentally measured to be $430 \mathrm{~mW}$, and the vision system we have used on previous capsules requires $180 \mathrm{~mW}$ for real-time streaming image transmission at video graphics array (VGA) resolution. Considering a 3.3 V supply, the average current required by the capsule under full load will be $184 \mathrm{~mA}$. This implies that a $100 \mathrm{~mA} \cdot \mathrm{h}$ battery would provide enough energy to complete an entire $30 \mathrm{~min}$ colon transit.

Note, however, that this calculation is somewhat conservative, since peristalsis will assist the capsule's motion, and may even be intentionally harnessed during portions of the journey that are not of interest to the desired diagnostic procedure. Commercial batteries having a nominal capacity of $120 \mathrm{~mA} \cdot \mathrm{h}$ would provide a margin of safety and the ability to capture additional images of other parts of the GI tract. The smallest examples of such batteries (e.g., the TLM-1030 from Tadiran, Israel) are generally approximately the same size as the capsule itself $(10 \mathrm{~mm}$ diameter by $30 \mathrm{~mm}$ long). Such a battery is encapsulated in a trailing plastic module shown attached to the capsule in Fig. 1. Whether this bimodular power solution will be accepted by both the medical community and patients themselves is an important issue that the authors are currently carefully evaluating.

Another promising potential solution is wireless power delivery, as described in [31]. This approach could be implemented by using an external coil together with three small coils integrated inside the locomotion module. A continuous energy transfer can be used to feed the different subsystems or can be stored onboard in a rechargeable battery or a capacitor.

\section{CONCLUSION}

While some miniaturization and power supply challenges remain in capsule robot design as discussed earlier, the mechanical locomotion module design, fabrication, and gait analysis results reported in this paper represent important steps toward a practical clinical legged capsule robot. Our lead screw/slot-follower mechanism enables miniaturization of the mechanical components of a legged capsule robot toward a swallowable size. We have also simultaneously added additional legs that are useful for tissue distention, and which we expect to enable the capsule to traverse flexures in the colon. The gait pattern we describe also enables the capsule to achieve higher speeds than prior designs while maintaining quality of locomotion. These speeds appear fast enough to enable the capsule to perform an inspection of the colon in a time period equivalent to current colonoscopy.

Replacing GI endoscopy with a swallowable capsule holds promise for improving patient comfort and safety during inspection of the colon, thus potentially enabling more effective screening of the GI tract for a larger number of patients. Swallowable capsules may also increase the number of people in the targeted screening group who undergo screening, by reducing indignity and discomfort.

\section{ACKNOWLEDGMENT}

The authors would like to thank novineon Healthcare Technology Partners GmbH, Tübingen, Germany, for assistance during the testing phase of the device. The magnetic encoder was developed by Sensitec GmbH within the framework of the VECTOR European Project. The authors are also grateful to N. Funaro, C. Filippeschi, and G. Favati for prototype manufacture, as well as E. Susilo, E. Buselli, S. Scapellato, and M. Domenichini for invaluable technical support.

\section{REFERENCES}

[1] A. Cuschieri, "Minimally invasive surgery: Hepatobiliary-pancreatic and foregut," Endoscopy, vol. 32, no. 4, pp. 331-344, Apr. 2000.

[2] B. V. MacFadyen and A. Cuschieri, "Endoluminal surgery," Surg. Endosc., vol. 19, no. 1, pp. 1-3, Jan. 2005.

[3] S. Schostek, H. Fischer, D. Kalanovic, and M. O. Schurr, "Microsystems in medicine-Results of an international survey," Minim. Invasive Ther. Allied Technol., vol. 14, no. 6, pp. 360-368, 2005.

[4] P. Dario, B. Hannaford, and A. Menciassi, "Smart surgical tools and augmenting devices," IEEE Trans. Robot. Autom., vol. 19, no. 5, pp. 782 792, Oct. 2003.

[5] G. Iddan, G. Meron, A. Glukhovsky, and P. Swain, "Wireless capsule endoscopy," Nature, vol. 405, no. 6785, p. 417, May 2000.

[6] G. D. Meron, "The development of the swallowable video capsule (M2A)," Gastrointest. Endosc., vol. 52, no. 6, pp. 817-819, Dec. 2000.

[7] L. Phee, A. Menciassi, S. Gorini, G. Pernorio, A. Arena, and P. Dario, "An innovative locomotion principle for minirobots moving in the gastrointestinal tract," in Proc. IEEE Int. Conf. Robot. Autom., 2002, vol. 2, pp. $1125-1130$.

[8] P. Breedveld, "Development of a rolling stent endoscope," in Proc. First IEEE/RAS-EMBS Int. Conf. Biomed. Robot. Biomechatron. BioRob.'06, pp. 921-926.

[9] B. Kim, H.-Y. Lim, K.-D. Kim, Y. Jeong, and J.-O. Park, "A locomotive mechanism for a robotic colonoscope," in Proc. IEEE/RSJ Int. Conf. Intell. Robots Syst., 2002, vol. 2, pp. 1373-1378.

[10] A. Moglia, A. Menciassi, M. Schurr, and P. Dario, "Wireless capsule endoscopy: From diagnostic devices to multipurpose robotic systems," Biomed. Microdevices, vol. 9, no. 2, pp. 235-243, Apr. 2007.

[11] A. Menciassi, M. Quirini, and P. Dario, "Microrobotics for future gastrointestinal endoscopy," Minim. Invasive Ther Allied Technol., vol. 16, no. 2, pp. 91-100, 2007.

[12] F. Carpi, S. Galbiati, and A. Carpi, "Controlled navigation of endoscopic capsules: Concept and preliminary experimental investigations," IEEE Trans. Biomed. Eng., vol. 54, no. 11, pp. 2028-2036, Nov. 2007.

[13] Olympus Endocapsule. [Online]. Available: www.olympus-europa.com/ endoscopy/

[14] H. Park, S. Park, E. Yoon, B. Kim, J. Park, and S. Park, "Paddling based microrobot for capsule endoscopes," in Proc. Robot. Autom.'07 IEEE Int. Conf., Apr. 10-14, 2007, pp. 3377-3382.

[15] M. Karagozler, E. Cheung, J. Kwon, and M. Sitti, "Miniature endoscopic capsule robot using biomimetic micro-patterned adhesives," in Proc. Biomed. Robot. Biomechatron.'06. BioRob'06. First IEEE/RAS-EMBS Int. Conf., Feb. 20-22, pp. 105-111.

[16] P. Dario, P. Ciarletta, A. Menciassi, and B. Kim, "Modelling and experimental validation of the locomotion of endoscopic robots in the colon," Int. J. Robot. Res., vol. 23, no. 4-5, pp. 549-559, 2004. 
[17] A. Menciassi, C. Stefanini, S. Gorini, G. Pemorio, P. Dario, B. Kim, and J. Park, "Legged locomotion in the gastrointestinal tract," in Proc. IEEE/RSJ Int. Conf. Intell. Robots Syst., 2004, vol. 1, pp. 937-942.

[18] M. Quirini, A. Menciassi, S. Scapellato, C. Stefanini, and P. Dario, "Design and fabrication of a motor legged capsule for the active exploration of the gastrointestinal tract," IEEE/ASME Trans. Mechatronics, vol. 13, no. 2, pp. 169-179, Apr. 2008.

[19] M. Quirini, S. Scapellato, A. Menciassi, P. Dario, F. Rieber, C. N. Ho, S. Schostek, and M. O. Schurr, "Feasibility proof of a legged locomotion capsule for the gi tract," Gastrointest. Endosc., vol. 67, no. 7, pp. 1153$1158,2008$.

[20] M. Quirini, R. Webster, A. Menciassi, and P. Dario, "Design of a pill-sized 12-legged endoscopic capsule robot," in Proc. Robot. Autom.'07 IEEE Int. Conf., Apr. 10-14, 2007, pp. 1856-1862.

[21] M. Quirini, R. J. Webster, A. Menciassi, and P. Dario, "Teleoperated endoscopic capsule," PCT Patent IT2007/000 259, 2007.

[22] R. Sidhu, D. S. Sanders, and M. E. McAlindon, "Gastrointestinal capsule endoscopy: From tertiary centres to primary care," BMJ (Brit. Med. J.), vol. 332, pp. 528-531, 2006.

[23] [Online]. Available: http://www.askasge.org/

[24] E. Buselli, P. Valdastri, M. Quirini, A. Menciassi, and P. Dario, "Superelastic leg design optimization for an endoscopic capsule with active locomotion," Smart Mater. Struct., vol. 18, no. 1, pp. 015001-1-015001-8, 2009.

[25] C. Stefanini, A. Menciassi, and P. Dario, "Modeling and experiments on a legged microrobot locomoting in a tubular, compliant and slippery environment," Int. J. Robot. Res., vol. 25, no. 5-6, pp. 551-560, 2006.

[26] [Online]. Available: http://www.roymech.co.uk/Useful_Tables/Tribology/ co_of_frict.htm

[27] E. C. Lee, "BEMF crossing detection in PWM mode operation for sensorless motor control application," U.S. Patent 5789 895, 1998, [Online]. Available: http://www.freepatentsonline.com/5789895.html

[28] E. Susilo, P. Valdastri, A. Menciassi, and P. Dario, "A miniaturized wireless control platform for robotic capsular endoscopy using pseudokernel approach," in Proc. XXII Eurosens. Conf., 2008, p. 490.

[29] M. M. Swindle, A. C. Smith, and B. J. Hepburn, "Swine as models in experimental surgery," J. Invest. Surg., vol. 1, no. 1, pp. 65-79, 1988.

[30] The ARES (Assembling Reconfigurable Endoluminal Surgical System) project website. [Online]. Available: http://www.ares-nest.org/

[31] B. Lenaerts and R. Puers, "An inductive power link for a wireless endoscope," Biosens. Bioelectron., vol. 22, pp. 1390-1395, 2007.

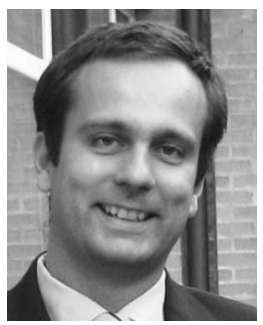

Pietro Valdastri (M'05) received the Laurea degree in electronic engineering (honors) from the University of Pisa, Pisa, Italy, in 2002, and the Ph.D. degree in bioengineering from Scuola Superiore Sant'Anna, Pisa, in 2006.

$\mathrm{He}$ is currently engaged in several European projects for the development of minimally invasive and wireless biomedical devices. He is currently an Assistant Professor of biomedical robotics at the Scuola Superiore Sant'Anna, where he is involved in research on implantable robotic systems and ac-

tive capsular endoscopy.

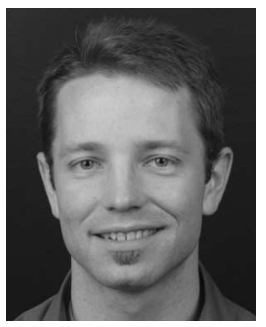

Robert J. Webster, III (S'97-M'08) received the B.S. degree in electrical engineering from Clemson University, Clemson, SC, in 2002, and the M.S. and $\mathrm{Ph} . \mathrm{D}$. degrees in mechanical engineering from the Johns Hopkins University, Baltimore, MD, in 2004 and 2007 , supported by a National Science Foundation Fellowship and a National Defense Science and Engineering Graduate Fellowship.

He joined the faculty of Vanderbilt University, Nashville, TN, in 2008, as an Assistant Professor in mechanical engineering, where he is currently the Director of the Medical and Electromechanical Design (MED) Laboratory. His current research interests include medical robotics, image-guided surgery, human-machine interfaces, and continuum robotics.

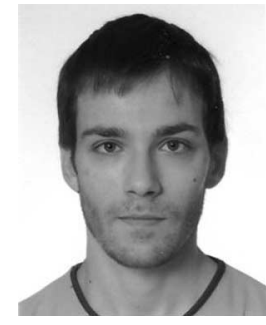

Claudio Quaglia received the Laurea degree in mechanical engineering from the University of Pisa, Pisa, Italy, in 2005 . He is currently working toward the Ph.D. degree in bioengineering at Scuola Superiore Sant'Anna, Pisa.

He was engaged in the employment of a triaxial accelerometer for tool condition monitoring in automatic machining. His current research interests include the field of medical robotics and micromechanical systems.

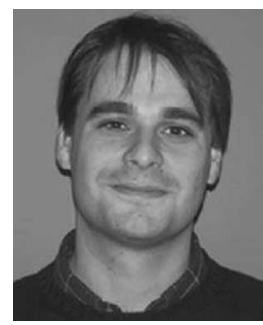

Marco Quirini (S'06) received the Laurea degree in mechanical engineering from the University of Pisa, Pisa, Italy, in 2004, and the Ph.D. degree from the Scuola Superiore Sant'Anna, Pisa, in 2009.

$\mathrm{He}$ is currently with the Scuola Superiore Sant'Anna. His current research interests include the fields of biomedical microrobotics, microfabrication technologies, micromechatronics, and microsystem technologies.

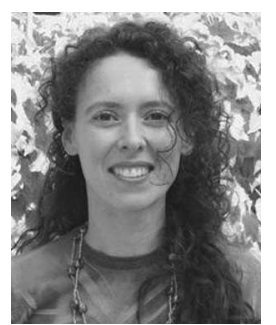

Arianna Menciassi (M'02) received the Master's degree in physics from the University of Pisa, Pisa, Italy, and the Ph.D. degree from the Scuola Superiore Sant'Anna, Pisa.

She is currently an Associate Professor of biomedical robotics at Scuola Superiore Sant'Anna.

Her current research interests include biomedical micro- and nanorobotics. She is the coauthor of about 100 international papers, about 50 ISI journals, and five book chapters on medical devices and microtechnologies.

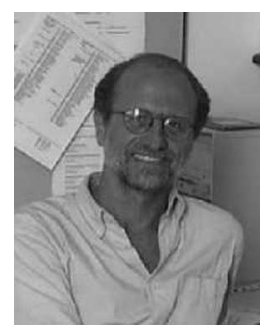

medical robotics.

Prof. Dario is a recipient of the Joseph Engelberger Award as a Pioneer of Biomedical Robotics. 